\title{
A Processing Workflow for Ocean Bottom Cable Dual-Sensor Acquisition Data: A Case of 3D Seismic Data in Bohai Bay
}

\author{
Xiaoming Yang, Yanchun Wang \\ School of Geophysics and Information Technology, China University of Geosciences, Beijing, China \\ Email: gnayxiao@163.com
}

How to cite this paper: Yang, X.M. and Wang, Y.C. (2019) A Processing Workflow for Ocean Bottom Cable Dual-Sensor Acquisition Data: A Case of 3D Seismic Data in Bohai Bay. Open Journal of Geology, 9 , 67-74

https://doi.org/10.4236/ojg.2019.91006

Received: January 2, 2019

Accepted: January 28, 2019

Published: January 31, 2019

Copyright $\odot 2019$ by author(s) and Scientific Research Publishing Inc. This work is licensed under the Creative Commons Attribution International License (CC BY 4.0).

http://creativecommons.org/licenses/by/4.0/

\begin{abstract}
The technique of ocean bottom cable (OBC) dual-sensor acquisition is an effective method to suppress the ghost wave and the reverberation at the receiver. With the advent of this technique, the processing method has become the key to the effective use of the OBC dual-sensor data. This paper has developed a new set of processing workflow based on the principle of combining the hydrophone and geophone data. This new process was applied to the OBC data acquired in Bohai area. The actual processing results show that the ghost and the reverberation are attenuated effectively. The frequency energy of the first notch point of the hydrophone data increased from $-22 \mathrm{~dB}$ to -13 $\mathrm{dB}$, and the frequency energy of the first notch point of the geophone data increased from $-18 \mathrm{~dB}$ to $-10 \mathrm{~dB}$. The spectral characteristics of the dual-sensor data are more reasonable. The frequency spectrum is broadened and richer, and the resolution of the stack profile is improved greatly.
\end{abstract}

\section{Keywords}

Ocean Bottom Cable (OBC), Ghost, Frequency Spectrum Notch, Reverberation, Sea Bottom Reflection Coefficient, Summing

\section{Introduction}

In seismic exploration, 3D seismic data describe reservoirs more accurately and guide production more effectively. For a long time, the towed streamer acquisition is a main method in 3D marine seismic exploration. As marine exploration improves day by day, there are many obstacles such as production-related platforms, marine traffic, and so on. These obstacles lead to huge gaps which reduce the number of coverage and ultimately affect the imaging results in towed 
streamer seismic surveys. In this case, the OBC method which employs a stationary array of receiver stations on the ocean bottom and a marine vessel towing only a seismic energy source becomes a natural choice.

There are some problems in the seismic data acquired by ocean bottom cable. It is well known that the sea surface and the seabed are two strong reflection interfaces. So when the seismic wave travels from the ground up to the sea, it continues to reflect downwards to the bottom of the sea, this process repeats. These interferences which disturb effective signal and generate frequency notches are called receiver-side free-surface multiples. These multiples, or "ghosts", have a worse effect in the shallow sea. As an example, if the water depth is $20 \mathrm{~m}$ and the wave speed is $1480 \mathrm{~m} / \mathrm{s}$, the wave field recorded by hydrophone receives a first frequency notch at $37 \mathrm{~Hz}$ (the first frequency notch of the hydrophone data is equal to the water sonic speed divided by 2 times the water depth) [1] [2] [3] [4]. The notch means that the seismic energy at certain frequency is cancelled by the interference of the multiple. In shallow water, the frequency components of these notches are within the effective frequency band. These interferences significantly reduce the bandwidth and resolution of the data. Obviously, eliminating "ghost" is the key to use the OBC data effectively. In seismic processing, predictive deconvolution is a common method to suppress the marine multiples. Unfortunately, the ghost filter recorded by the hydrophone is mixed phase, and ghost filter recorded by the geophone is minimum phase. Therefore, predictive deconvolution cannot be used to eliminate the ghost.

Based on a simple observation that the ghost waves recorded by hydrophone and geophone have an opposite polarity, geophysicists propose an OBC dual-sensor acquisition technique to eliminate ghost waves. Barr and Sanders (1989) propose that properly combining the hydrophone and geophone data sets can eliminate not only the receiver ghost, but also all water-column reverberations (at the receiver) [5]. Ralph, Sanders, and Starr (1993) prove that the characters of the OBC dual-sensor data are consistent with the characters of streamer data. Basically, the quality of the OBC dual-sensor data is better than that of the streamer data [6]. So far, the OBC dual-sensor acquisition technique has been widely used in marine exploration.

In the OBC dual-sensor processing, Barr and Sanders (1989) places special calibration shots to record the direct arrivals and then analysis the scale factor at each detection point [7] [8]. This method obtains accurate scale factors, but it will add more cost of a survey. Bill Dragoset and Fred J. Barr (1994) derive calibration scale factors from the data directly. This method based on the criterion that the proper scale factors are those that best whiten the summed data [9]. Hoffe (2000) described the wave field characteristics of OBC dual-sensor seismic data and revealed the physical nature of dual-sensor data summing [10]; Soudani (2006) proposed a three-dimensional OBC combining processing techniques [11]; Hugonnet (2011) presented a three-step OBC dual-sensor combining processing technique by using the cross ghost wave operator [12]. Zhang (2015) studied the method of OBC seismic data with low SNR [13]. The above research 
has studied the method of $\mathrm{OBC}$ dual-sensor combining in theory or from a specific step, and does not list detailed processing workflow for actual data.

This paper proposes a multi-domain processing workflow for the $\mathrm{OBC}$ dual-sensor acquisition data. In order to obtain the best scale factor, this workflow is more careful and detailed in noise attenuation, and the noise is suppressed in multi-domains. We applied the workflow to an OBC dual-sensor data acquired in 2011 from Bohai Bay. The results show that this processing workflow greatly improves the quality of seismic data.

\section{Method}

In OBC dual-sensor survey, the mechanism of receiving signals by hydrophone and geophone is different. The signal recorded by the hydrophone is a pressure scalar, and the polarity of a scalar response is independent of the direction of propagation. The geophone detects the velocity of the particles which is a vector response. The polarity of a vector response is related to the direction of propagation. When the seismic wave propagates from the sea surface to the sea floor, it is a down wave signal; when the seismic wave is reflected from the ground layer to the sea bottom, it is an up wave signal. Figure 1 shows that the hydrophone and the geophone receive the same polarity of up-going wave and opposite polarity down-going wave. Summing the dual-sensor recording can only eliminate the down-going wave, some up-going reverberation still exist in the result.

Suppose that the depth of the receiver and the refection coefficient of the seabed are known, after some mathematical operations, the OBC dual-sensor combination can suppress all the reverberation at the receiver (Figure 2). When the primary wave reaches the bottom of the sea from underground, assuming that the refection coefficient of the seabed is $r$, the primary wave is $x(t)$, the two-way travel of seismic wave is $\tau$, the wave field recorded by hydrophone is $G(t)$, the wave field recorded by geophone is $H(t)$. The seismic wave field recorded by hydrophone and geophone can be expressed as the following formula:

The wave field of hydrophone

$$
\begin{aligned}
H(t) & =(1+r) x(t)-(1+r)^{2} x(t-\tau)+(1+r)^{2} r x(t-2 \tau)+\cdots \\
& =x(t)-(1+r) \sum_{i=1}^{\infty}(-r)^{i-1} x(t-i \tau)
\end{aligned}
$$

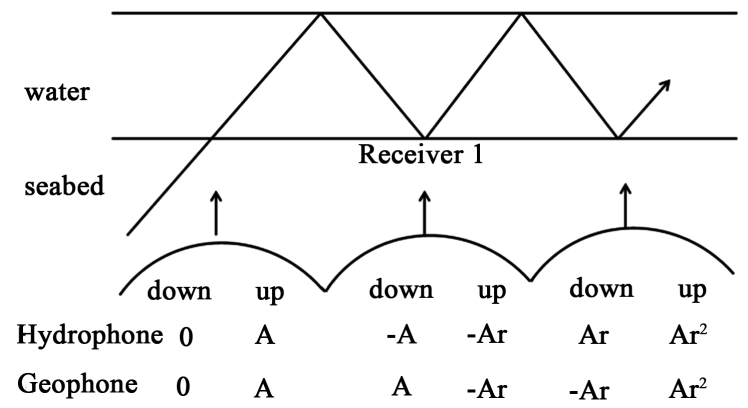

Figure 1. The propagation schematic of up-going and down-going wave. 


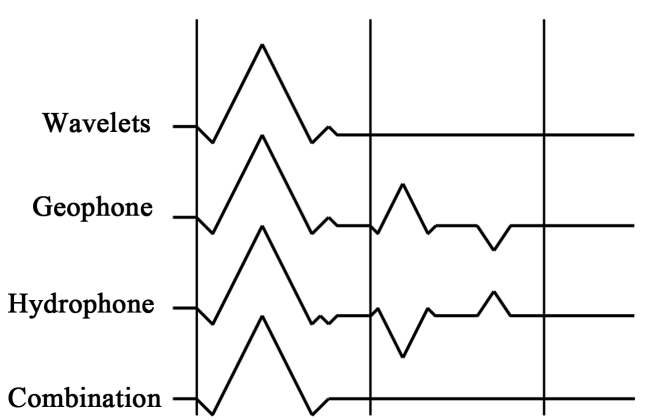

Figure 2. The principle of eliminating ghost by combining of the hydrophone and geophone data.

The wave field of geophone

$$
\begin{aligned}
G(t) & =(1-r) x(t)+(1-r)^{2} x(t-\tau)-(1-r)^{2} r x(t-2 \tau)+\cdots \\
& =x(t)+(1-r) \sum_{i=1}^{\infty}(-r)^{i-1} x(t-i \tau)
\end{aligned}
$$

If we multiply the geophone by a proportional coefficient $k=\frac{1+r}{1-r} \quad(0<r<1)$, and add it with the hydrophone data (Formula (3)), we can get a seismic record in which the reverberation at the receiver is eliminated completely.

$$
\text { Sum }=H(t)+\frac{1+r}{1-r} G(t)=\frac{2}{1-r} x(t)
$$

The key of summing is the calculation of the accurate reflection coefficient of seabed or the scale factors. Our method of extracting scale factors from seismic data is based on finding scalar that best whiten the summed data. This method needs minimizing the impact of noise as much as possible. Therefore, the noise in $\mathrm{OBC}$ data must be suppressed properly before combination processing.

In fact, hydrophone and geophone have different sensitivities to the various kinds of noises that can be present in the ocean-bottom environment. The appropriate method is taken to attenuate the noise between hydrophone and geophone data in the shot domain, common middle point (CMP) domain, common reflection point (CRP) domain, etc. The processing workflow for OBC dual-sensor acquisition data is shown as Figure 3.

\section{Example}

This processing workflow was applied to $3 \mathrm{D}$ OBC dual-sensor seismic data acquired in the Bohai Bay Region. In this survey, the depth of the water is $2-20 \mathrm{~m}$. The source-line spacing and shot-point interval are $100 \times 50 \mathrm{~m}$, with receiver-line spacing and station interval $200 \times 50 \mathrm{~m}$. The recording time is $8 \mathrm{~s}$, the sample interval is $1 \mathrm{~ms}$.

Figure 4 shows the noise attenuation processing of geophone data in shot domain. Figure 4(a) is a raw shot gather. After noise attenuation, the noise was suppressed greatly and the signal to noise was improved obviously (Figure 4(b)). Figure $4(c)$ is the noise, from which we can see that effective waves are 


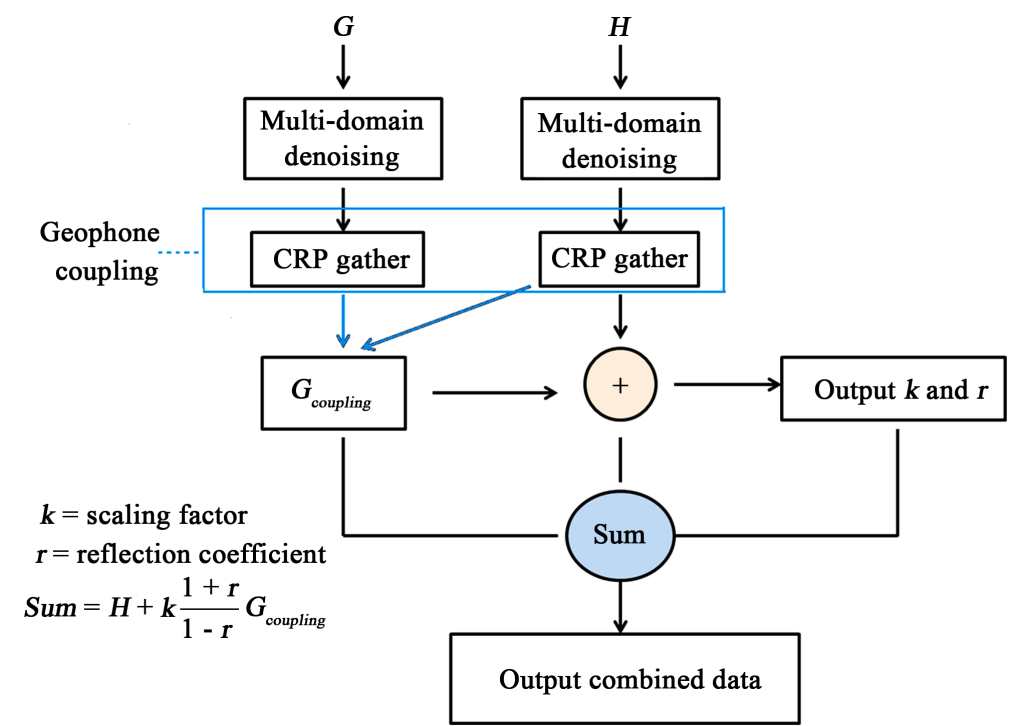

Figure 3. The processing workflow for OBC dual-sensor acquisition data.

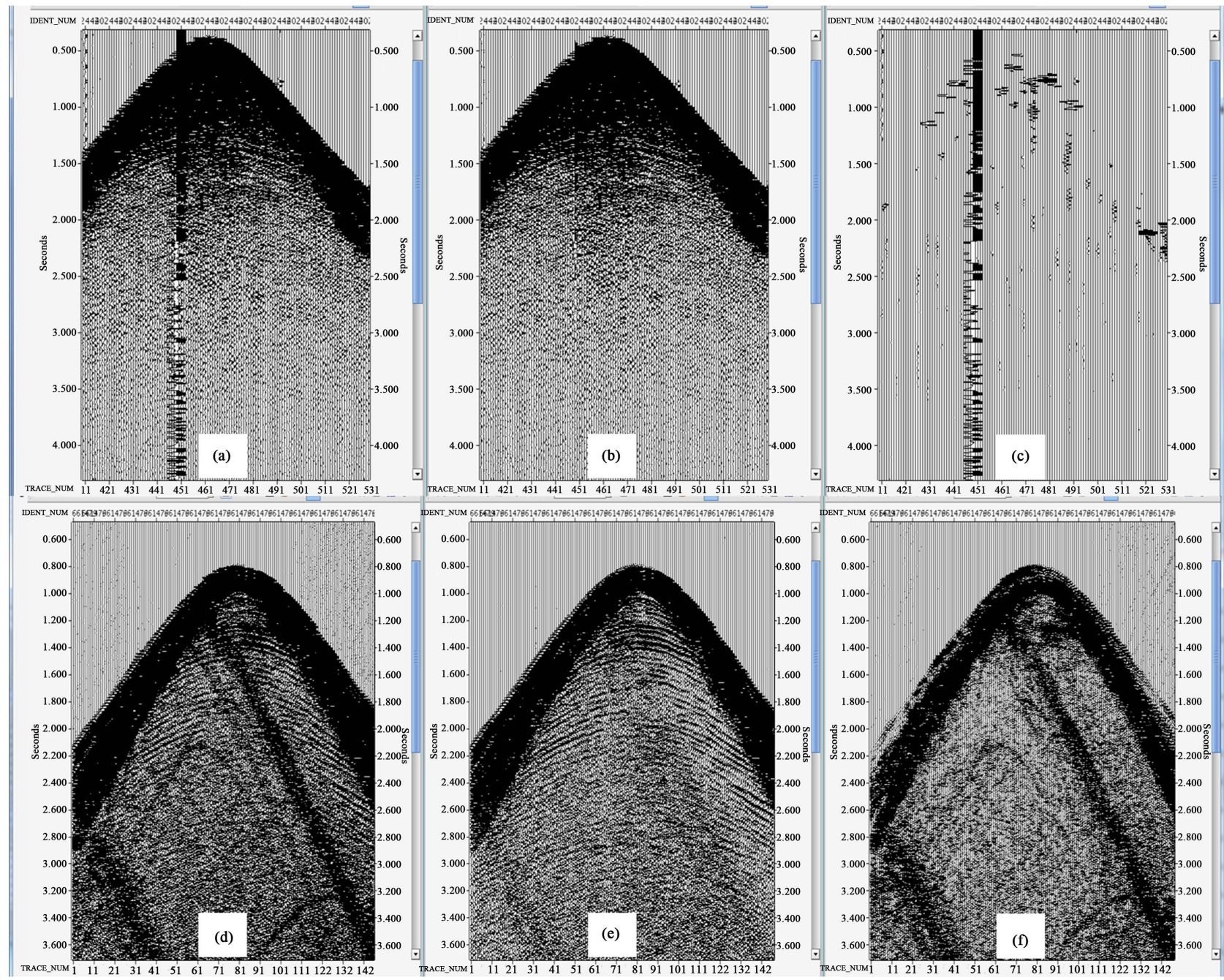

Figure 4. Geophone shot gathers. Gather before noise attenuation (a); gather after noise attenuation (b); and the noise removed (c); Noise attenuation of hydrophone shot gathers ((d)-(f)). 
well protected. Figures $4(\mathrm{~d})$-(f) shows the noise attenuation processing of hydrophone data in shot domain. The results are as good as that of the geophone data.

Figure 5(a) shows the significant linear interference in the CRP domain which appears as random noise in other domains. This interference makes the calculation of the scale factor unreliable. After noise attenuation (Figure 5(b)), the quality profile has been greatly improved, and the effective waves are clearly visible. Figure $5(\mathrm{c})$ is the noise eliminated.

Figure 6 shows the comparison of CMP stack between before and after dual-sensor combination. After dual-sensor combination, the event is clearer.

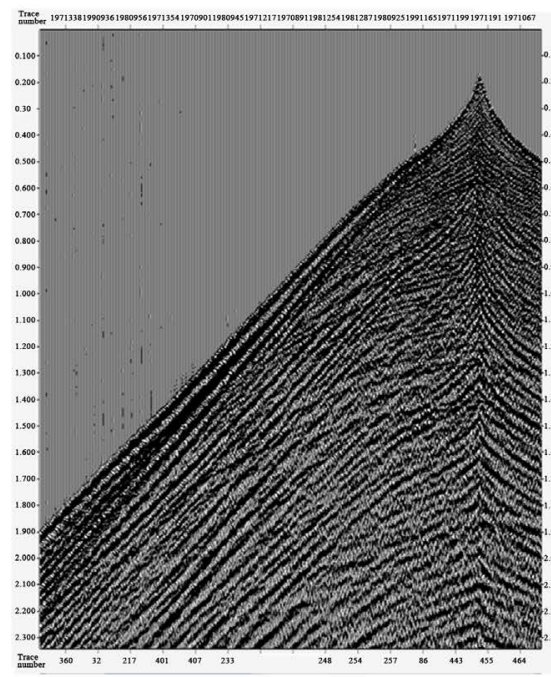

(a)

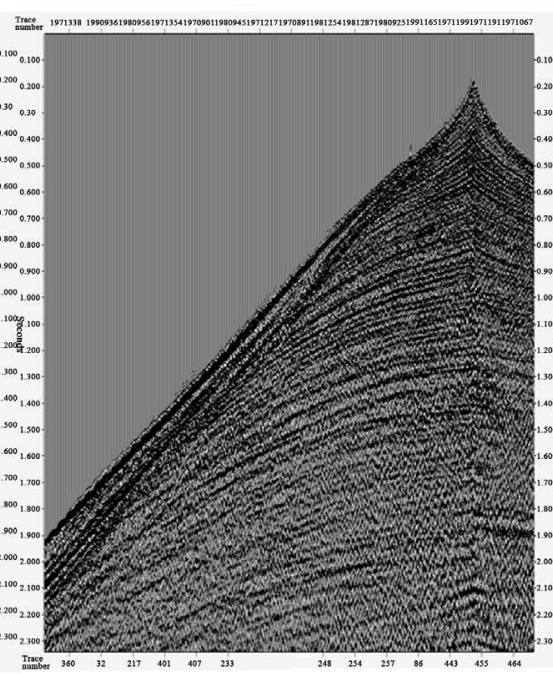

(b)

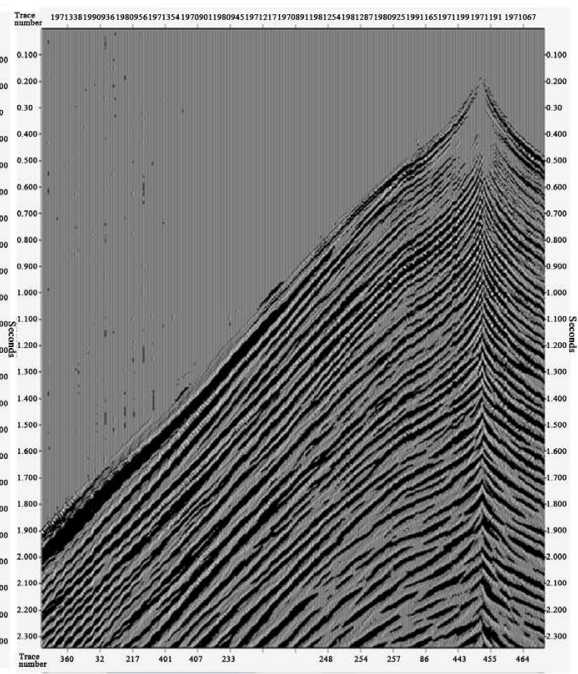

(c)

Figure 5. Geophone CRP gathers. Gather before noise attenuation (a); gather after noise attenuation (b); and the noise removed (c).

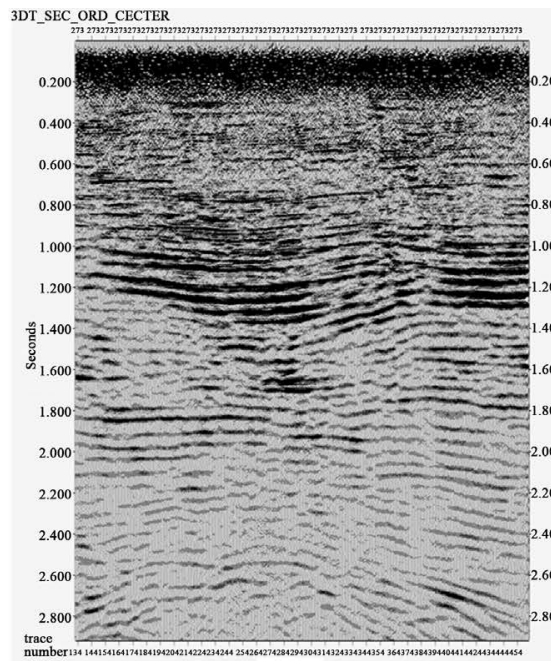

(a)

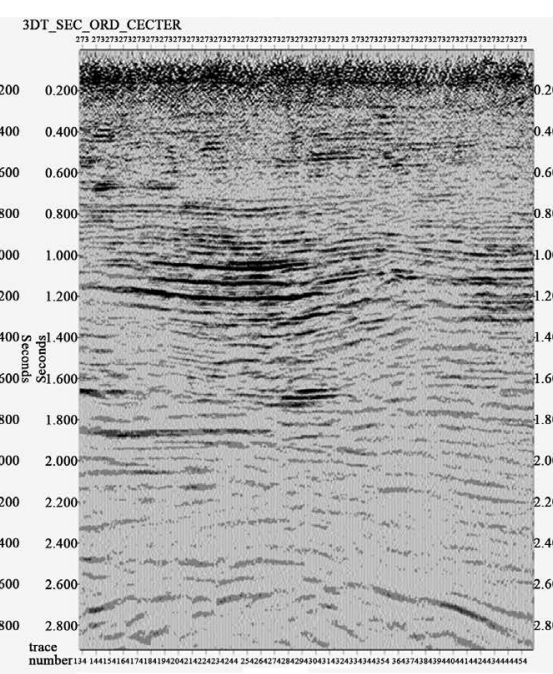

(b)

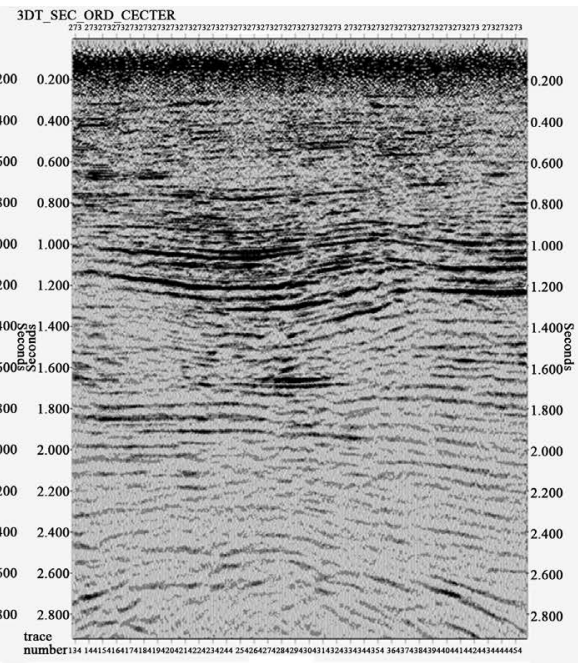

(c)

Figure 6. Comparison of CMP stack between before and after dual-sensor combination. The stack of hydrophone data (a); the stack of geophone data (b); the stack of dual-sensor combination (c). 
Figure 7 shows the comparison of spectrum between before and after dual-sensor combination. The notches in the spectrum of hydrophone and geophone data are both caused by reverberation. After the processing of combination, the frequency energy of the first notch point of the hydrophone data increased from $-22 \mathrm{~dB}$ to $-13 \mathrm{~dB}$, and the frequency energy of the first notch point of the geophone data increased from $-18 \mathrm{~dB}$ to $-10 \mathrm{~dB}$. The spectral characteristics of the dual-sensor data are more reasonable.

As shown in Figure 8, before dual-sensor combination, the side lobes energy of both hydrophone and geophone data is very obvious. After the combination processing, the reverberation is eliminated, and the side lobe energy that represents multiple waves is greatly suppressed.

\section{Conclusions}

About the dual-sensor OBC data processing, we can come to conclusions:

1) The presence of noise affects the accuracy of the scale factor. So it is necessary to perform noise attenuation in multi-domain.

2) In order to ensure that the opposite polarity noise in the dual-sensor data can exactly cancel each other, each processing step that affects multiple waves in the data should be used with caution. Before combination, the deconvolution processing must not be applied.

3) Dual-sensor OBC combination processing can remove notch, broaden frequency band and enrich the frequency spectrum.

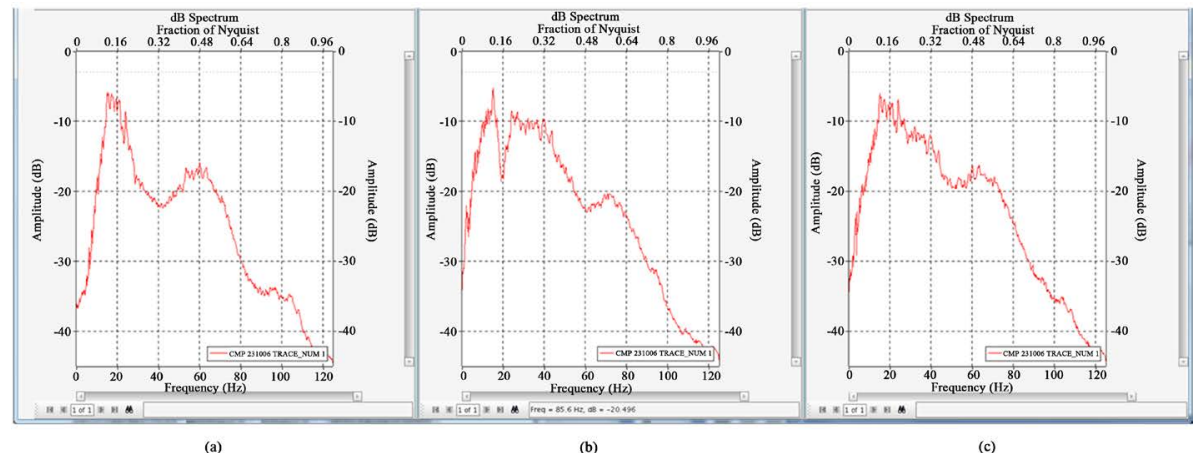

(c)

Figure 7. Comparison of spectrum between before and after dual-sensor combination. The spectrum of hydrophone data (a); the spectrum of geophone data (b); the spectrum of dual-sensor combination (c).

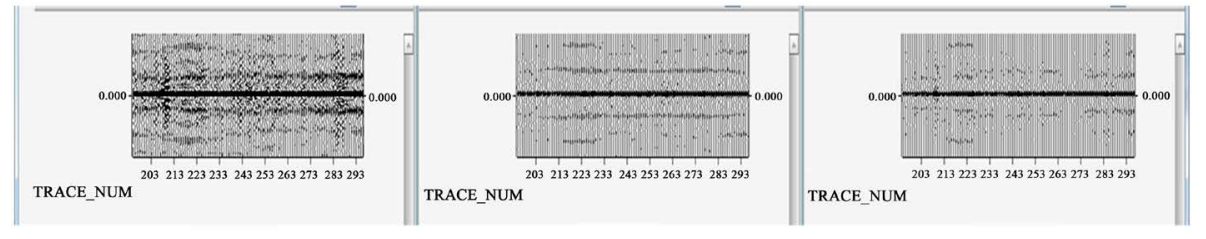

(a)

(c)

Figure 8. Comparison of autocorrelation between before and after dual-sensor combination. The autocorrelation of hydrophone data (a); the autocorrelation of geophone data (b); the autocorrelation of dual-sensor combination (c). 


\section{Conflicts of Interest}

The authors declare no conflicts of interest regarding the publication of this paper.

\section{References}

[1] Wang, D.K., Tong, S.Y., Liu, H.S. and Zhu, W.Q. (2015) Notch Effect and Frequency Compensation of Dual-Sensor OBC Data in Shallow Water. Journal of Earth Science, 26, 508-514. https://doi.org/10.1007/s12583-015-0559-2

[2] Soubaras, R. (1996) Ocean Bottom Hydrophone and Geophone Processing. SEG Technical Program Expanded Abstracts, 1996, 24-27.

[3] Gong, X.D. (2014) Influence of Water Depth Error of Detection Point on Combined Processing of OBC Double Inspection Data and Countermeasures. Geophysical Prospecting for Petroleum, 53, 324-329.

[4] Zhou, B., Gong, X., Gao, M. and Zhang, J. (2015) An Improvement of The Dual-Sensor Summation Technique for Cross-Ghosting from OBC and Its Application. China Offshore Oil and Gas, 27, 49-52.

[5] Barr, F.T. and Paffenholz, J. (1996) The Dual-Sensor Ocean-Bottom Cable Method: Comparative Geophysical Attributes, Quantitative Geophone Coupling Analysis and Other Recent Advances. SEG Technical Program Expanded Abstracts, 1996, 21-23.

[6] Ralph, J., Sanders, J.I. and Starr, J. (1993) Extending Offshore Limits with Joint Streamer and Ocean Bottom Cable Operations. SEG Technical Program Expanded Abstracts, 1993, 540-543.

[7] Barr, F.J. and Joe, I.S. (1989) Attenuation of Water-Column Reverberations Using Pressure and Velocity Detectors in a Water-Bottom Cable. SEG Technical Program Expanded Abstracts, 1989, 653-656.

[8] Barr, F.J. (1997) Dual-Sensor OBC Technology. The Leading Edge, 16, 45-52. https://doi.org/10.1190/1.1437427

[9] Dragoset, B., Fred, J. and Barr, F.J. (1994) Ocean-Bottom Cable Dual-Sensor Scaling. SEG Technical Program Expanded Abstracts, 1994, 857-860.

[10] Hoffe, B.H., Laurence, R., et al. (2000) Applications of OBC Recording. The Leading Edge, 19, 382-391. https://doi.org/10.1190/1.1438616

[11] Soudani, M.T.A, Boelle, J.L. and Hugonnet, P. (2006) 3D Methodology for OBC Pre-Processing. The 68th EAGE Conference \& Exhibition Incorporating SPE EUROPEC, Vienna, 12-14 June 2006, B044.

[12] Hugonnet, P., Boelle, J.L. and Herrmann, P. (2011) PZ Summation of 3D WAZ OBS Receiver Gathers. The 73rd EAGE Conference \& Exhibition Incorporating SPE EUROPEC, Vienna, 23-26 May 2011, B002. https://doi.org/10.3997/2214-4609.20148974

[13] Zhang, B.Q., Zhou, H.W., Ding, Z.Y., et al. (2015) Integrated Processing Techniques to Low Signal-to-Noise Ratio OBC Dual-Sensor Seismic Data. The 85th SEG Annual International Meeting, New Orleans, 18-23 October 2015, 2180-2184. https://doi.org/10.1190/segam2015-5860831.1 